\title{
A catalogue of emission lines in spectra of Comet C/1995 01 (Hale-Bopp) $^{\star}$
}

\author{
H. W. Zhang ${ }^{1,2,3}$, G. Zhao ${ }^{1}$, and J. Y. $\mathrm{Hu}^{1}$ \\ 1 Beijing Astronomical Observatory, Chinese Academy of Sciences, Beijing 100012, PR China \\ 2 Department of Astronomy, Peking University, Beijing 100871, PR China \\ 3 Beijing Astrophysics Center, Jointly sponsored by the Chinese Academy of Sciences and Peking University, \\ Beijing 100871, P.R. China
}

Received 29 June 2000 / Accepted 23 November 2000

\begin{abstract}
High-resolution and high signal-to-noise ratio spectra of Comet C/1995 O1 (Hale-Bopp), which provided continuous wavelength coverage from $5500 \AA$ to $8500 \AA$ and partial coverage from $4000 \AA$ to $5500 \AA$, were obtained with the Coudé Echelle Spectrograph at Beijing Astronomical Observatory on March 26, 28 and April 22, 1997. In the spectra we found 532 emission features, among which 459 lines from $\mathrm{H}, \mathrm{O}, \mathrm{Na}, \mathrm{C}_{2}, \mathrm{C}_{3}, \mathrm{CN}, \mathrm{CH}, \mathrm{NH}_{2}$ and $\mathrm{H}_{2} \mathrm{O}^{+}$were identified. The intensity of sodium emission lines at $5890 \AA$ and $5896 \AA$ on April 22 increased about 5 fold compared to that recorded on March 26 and 28 . The intensity ratio $I_{\lambda 5577} /\left(I_{\lambda 6300}+I_{\lambda 6364}\right)$ of [O I] is consistent with the formation of excited $\mathrm{O}$ atoms from the photodissociation of $\mathrm{H}_{2} \mathrm{O}$.
\end{abstract}

Key words. comets: general - comet: C/1995 O1 (Hale-Bopp)

\section{Introduction}

High-resolution spectral observation of Comet C/1995 O1 (Hale-Bopp) provides an opportunity to investigate the cometary chemical composition. It is significant that comets are the least modified remnants of the creation of the solar system and can provide insight into conditions existing in the early solar system. Low resolution optical spectra of many comets have been obtained (e.g. Hicks \& Fink 1996). Arpigny (1995) pointed out that some 200300 of the approximately 2000 emission lines in the optical region of cometary spectra have yet to be assigned as molecular species.

With echelle spectrographs, we can obtain high resolution and large wavelength coverage spectra in optical regions. Such high resolution optical spectra of comets can be used to identify new molecular species. For example, Brown et al. (1996) gave a catalogue with 2997 emission lines based on high resolution spectra of comet Swift-Tuttle and Brorsen-Metcalf over the range of 3800$9000 \AA$ A. They identified 2438 lines caused by $\mathrm{H}, \mathrm{O}, \mathrm{C}_{2}$, $\mathrm{CN}, \mathrm{NH}_{2}, \mathrm{C}_{3}, \mathrm{H}_{2} \mathrm{O}^{+}, \mathrm{CH}$ and $\mathrm{CH}^{+}$. Morrison et al.

Send offprint requests to: H. W. Zhang,

e-mail: zhw@bac.pku.edu.cn

* Table 3 is only available in electronic form at the CDS via anonymous ftp to cdsarc.u-strasbg.fr (130.79.128.5) or via

http://cdsweb.u-strasbg.fr/cgi-bin/qcat?J/A+A/367/1049
(1997) also obtained the high resolution spectra of Comet C/1996 B2 (Hyakutake). However, some emission lines found in comets are still unidentified. Although many of the unidentified features are likely to belong to alreadyknown radicals and ions, the existence of yet unknown molecules responsible for these emissions cannot be excluded. Therefore, it is important to verify the presence of these lines in more comets. Their variation from comet to comet might also be helpful in identification.

In this paper, we present a catalogue of emission lines from the high-resolution and high signal-to-noise ratio spectra of Comet C/1995 O1(Hale-Bopp). The observation and data reduction are described in Sect. 2. The line identifications are described in Sect. 3. A brief discussion of our results on $\mathrm{Na}$ and [O I] is given in Sect. 4 .

\section{Observation and data reduction}

The observation was made by using the Coudé Echelle Spectrograph mounted on the $2.16 \mathrm{~m}$ telescope at Beijing Astronomical Observatory (Xinglong, China). The detector was a Tek CCD $(1024 \times 1024$ pixels of $24 \mu \mathrm{m} \times 24 \mu \mathrm{m}$ each). The Coudé Echelle Spectrograph consists of a blue path and a red path. For the blue path, the 79 grooves $/ \mathrm{mm}$ echelle grating was used, along with a prism as cross disperser and a $0.5 \mathrm{~mm}\left(1.06^{\prime \prime}\right)$ slit leading to a resolving power of the order of 44000 . For the red path, the 31.6 grooves $/ \mathrm{mm}$ echelle grating was used, along with a 
Table 1. Journal of observations. The observation date, the universal time of mid-exposure, the exposure duration, the signalto-noise ratio on continuum at $6400 \AA$, the spectrum range, the geocentric and heliocentric distances $(\Delta, r)$ and the radial velocities $(\dot{\Delta}, \dot{r})$ of the comet interpolated to the time of mid-exposure are listed. The ephemeris for Comet C/1995 O1 (HaleBopp) was computed from the orbit solution \#55 published on March 4, 1997 by Yeomans and are available to the public via Internet (http://encke.jpl.nasa.gov/eph.html)

\begin{tabular}{ccccccccc}
\hline \hline Date & U.T. & $\begin{array}{c}\text { Exp. Time } \\
(\mathrm{min})\end{array}$ & $\begin{array}{c}S / N \\
\left(\mathrm{pixel}^{-1}\right)\end{array}$ & Sp. Range & $\begin{array}{c}\Delta \\
(\mathrm{AU})\end{array}$ & $\begin{array}{c}r \\
(\mathrm{AU})\end{array}$ & $\begin{array}{c}\dot{\Delta} \\
\left(\mathrm{km} \mathrm{s}^{-1}\right)\end{array}$ & $\begin{array}{c}\dot{r} \\
\left(\mathrm{~km} \mathrm{~s}^{-1}\right)\end{array}$ \\
\hline $26 / 03 / 1997$ & $11: 22: 45$ & 30 & 180 & Red & 1.322 & 0.920 & 5.6 & -3.5 \\
$28 / 03 / 1997$ & $11: 45: 42$ & 20 & 180 & Red & 1.330 & 0.918 & 8.3 & -1.8 \\
$28 / 03 / 1997$ & $12: 09: 54$ & 20 & 280 & Red & 1.330 & 0.918 & 8.3 & -1.8 \\
$22 / 04 / 1997$ & $11: 47: 55$ & 20 & 80 & Blue & 1.627 & 0.991 & 28.4 & 12.2 \\
\hline \hline
\end{tabular}

prism as cross disperser and a $0.5 \mathrm{~mm}\left(1.06^{\prime \prime}\right)$ slit leading to a resolving power of the order of 37000 . The wavelength coverage in one exposure is limited by CCD size.

The spectra of Comet C/1995 O1 (Hale-Bopp) were obtained on March 26, 28 and April 22, 1997 (see Table 1). The slit was positioned on the brightest point of the comet and manually guided on the comet. Four spectra were obtained during these observations. They provided continuous wavelength coverage from $5500 \AA$ to $8500 \AA$ and partial coverage from $4000 \AA$ to $5500 \AA$. The wavelength coverage of each spectrum is listed in Table 2 . The spectrum of the moon was observed on March 27.

The spectra were reduced with the ESO MIDAS package on a SUN Sparc 20 workstation at Beijing Astronomical Observatory (Beijing, China). The data reduction includes locating the echelle order on the multiorder two dimensional spectrum, subtracting the background, and extracting the orders by summation along the slit. The pixel-to-pixel variation was corrected by dividing by flat fields taken at the same night. The wavelength calibration was based on a thorium-argon lamp. The continuum level was determined by fitting a spline curve to a set of continuum window (typically $20-30$ per order). The continuum windows of spectra were selected by inspection of the solar atlas (Moore et al. 1966). The ranges of cometary emissions were excluded by checking sudden variations in intensity compared to intensities in neighbouring continuum windows. Division of the spectrum by the fitted curve yielded a normalized spectrum. Since the conditions were not photometric in both March and April, no accurate absolute calibration could be made. The spectral atlas with the wavelength range from $5500 \AA$ to $8500 \AA$ of Comet C/1995 O1 (Hale-Bopp) on 28 March was published by Zhang et al. (1997a,b).

\section{Line identification}

In order to distinguish emission features, moon spectra were subtracted. Both comet and moon spectra were shifted to the continuum at zero heliocentric radial velocity. A portion of the subtracted spectra on March 28 is shown in Fig. 1.

Emission features were searched for in all subtracted spectra, and every feature was cross-checked in several spectra covering the same wavelength range. Emission lines between $5500 \AA$ to $8500 \AA$, which were confirmed in different spectra, were included in our line catalogue. In addition, very strong and significant emission lines between $4000 \AA$ and $5500 \AA$ were selected. Regions around $6900 \AA$ and $7600 \AA$ in which water lines are rich were skipped because of spurious emission features at the locations of water lines in the subtracted spectra. In order to exclude the residuals of the continuum subtraction, the emission features were also checked in the unnormalized and unsubtracted spectra. Emission lines from the sky, primarily due to $\mathrm{OH}$, were identified and removed using the table of Osterbrock et al. (1996). The wavelengths and equivalent widths $(E W \mathrm{~s})$ of emission features were measured. The accuracy of the wavelength measurement is about $0.1 \AA$. Finally, 532 secure emission features were selected for our line catalogue. Weak features $(E W<5 \mathrm{~m} \AA)$ were excluded.

Our line identifications are based on wavelength coincidences only. The catalogue of Brown et al. (1996) was used as a starting line list. In total, we identified 459 of 532 emission features, which included $231 \mathrm{C}_{2}$ lines, $163 \mathrm{NH}_{2}$ lines, $45 \mathrm{CN}$ lines, $6 \mathrm{H}_{2} \mathrm{O}^{+}$lines, $7 \mathrm{CH}$ lines, $1 \mathrm{C}_{3}$ lines, 3 [O I] lines, $2 \mathrm{Na}$ lines and $1 \mathrm{H}$ line. Table 3 lists all of the lines found in spectra and their identifications, if known. An example of Table 3 is given here. The complete catalogue is provided only in electronic form at the CDS. Table 4 lists all 73 unidentified lines.

Figure 2 gives, in graphical form, the average wavelengths and $E W \mathrm{~s}$ of cometary emission lines between $5500 \AA$ and $8500 \AA$. The figure shows that $\mathrm{NH}_{2}$ lines are relatively strong and $\mathrm{C}_{2}, \mathrm{CN}$, and $\mathrm{H}_{2} \mathrm{O}^{+}$lines are quite weak. Probably, the reason is the differences in abundances and spatial distribution of the species in the comet.

Compared with Fig. 1b of Brown et al. (1996) and Fig. 1 of Morrison et al. (1997), Fig. 1a of this paper shows that $\mathrm{NH}_{2}$ and some unidentified lines are significantly stronger, relative to the $\mathrm{C}_{2}$ lines. This may be a clue to the origin of the unidentified lines. Considering wavelengths and relative strengths, we suspect that these unidentified lines are $\mathrm{NH}_{2}$ lines. However, the identification of these lines will be the subject of our forthcoming work. 

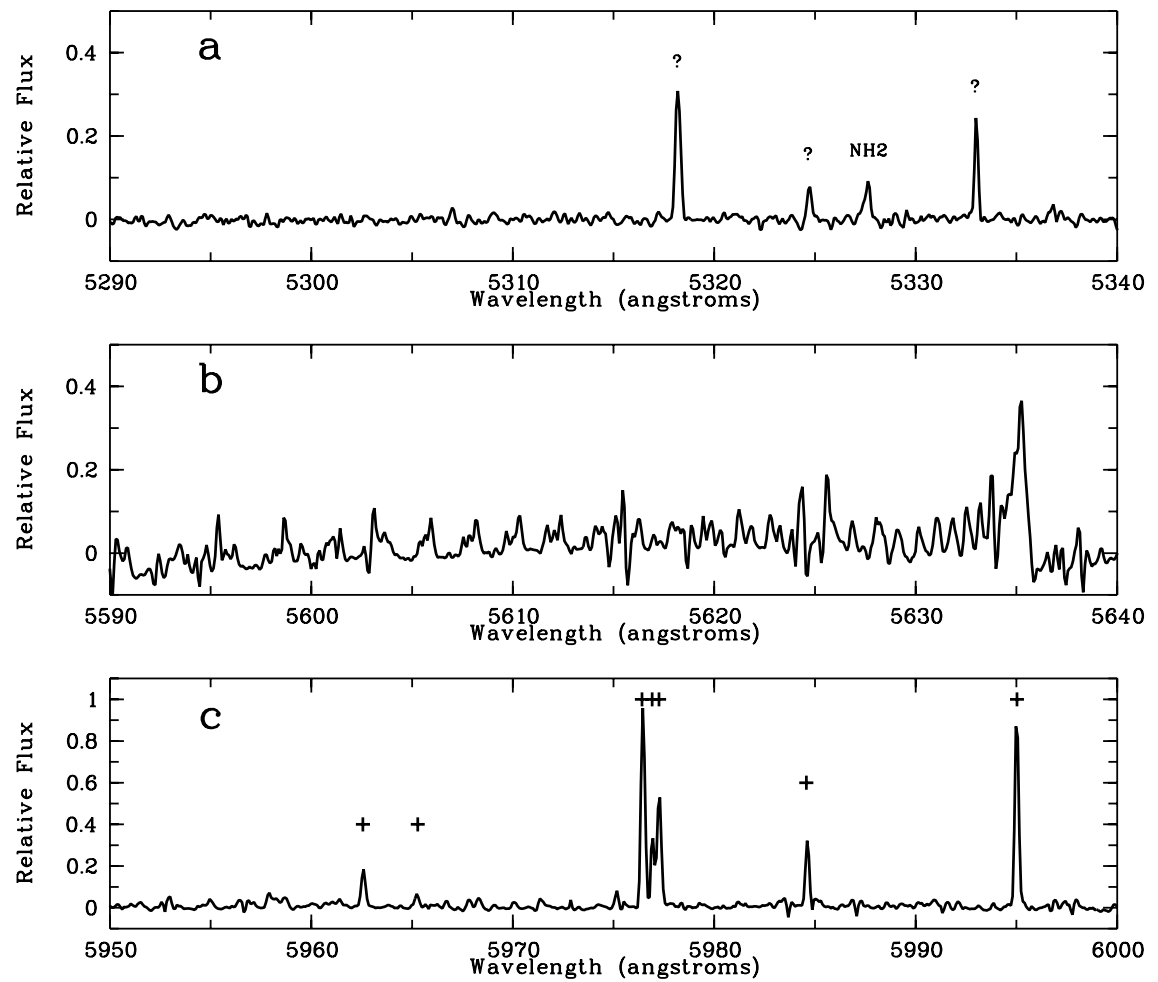

Fig. 1. A portion of comet spectra. a) A region showing unidentified lines marked with "?". b) A region showing the $\mathrm{C}_{2}$ band. c) A region showing $\mathrm{NH}_{2}$ lines marked with a cross

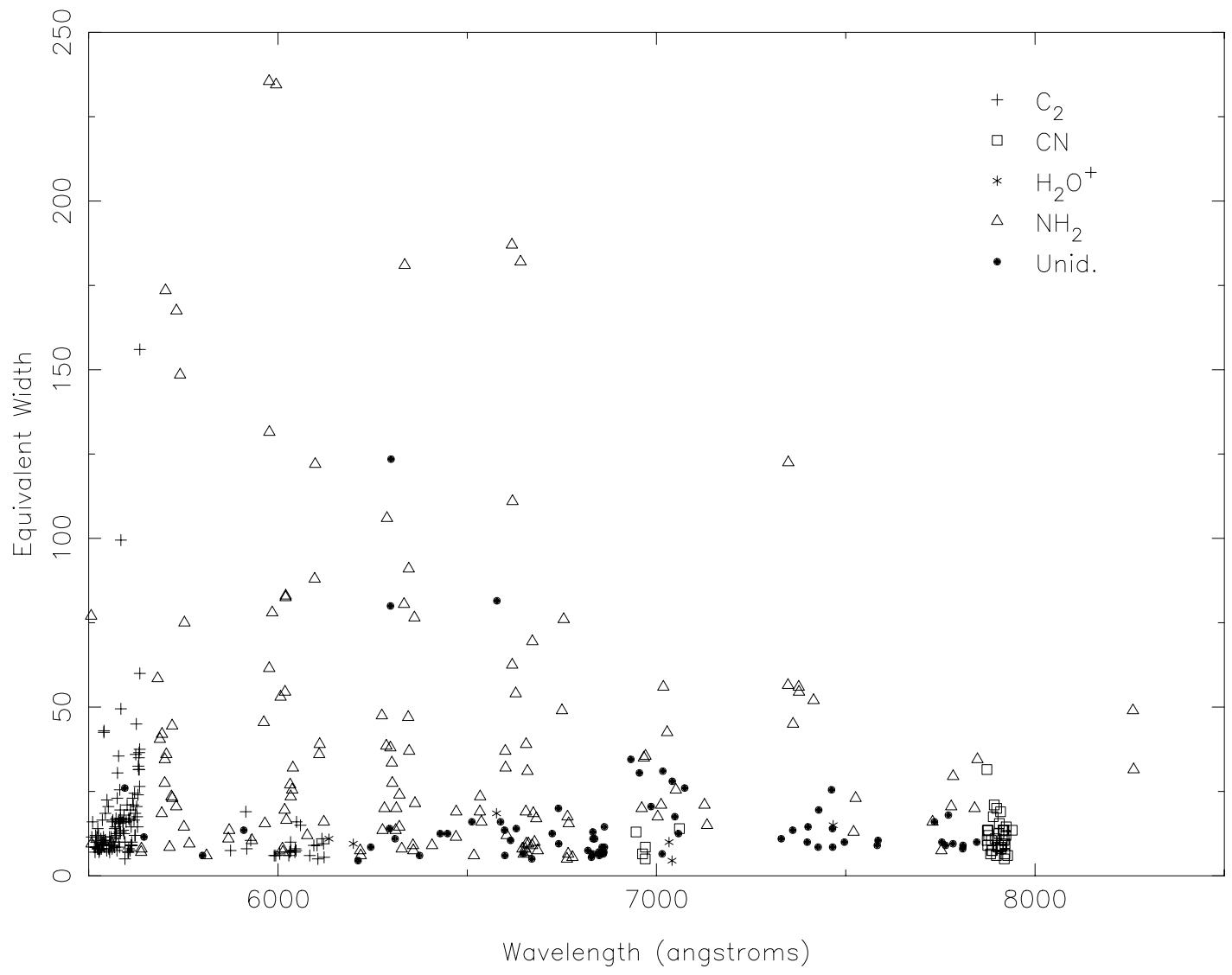

Fig. 2. Emission lines found in spectra between $5500 \AA$ and $8500 \AA$; unit of equivalent width is $\mathrm{m} \AA$ 
Table 2. Wavelength coverage of the echelle spectra

\begin{tabular}{|c|c|c|c|c|c|}
\hline Echelle order & 26 March & 28 March & $28 \mathrm{March}$ & Echelle order & 22 April \\
\hline 66 & $8498-8617$ & & & 35 & $6344-6423$ \\
\hline 67 & $8372-8489$ & $8406-8521$ & $8456-8577$ & 36 & $6168-6245$ \\
\hline 68 & $8248-8364$ & $8283-8396$ & $8330-8450$ & 37 & $6001-6076$ \\
\hline 69 & $8127-8240$ & $8162-8276$ & $8208-8325$ & 38 & 5843-5916 \\
\hline 70 & $8013-8125$ & $8046-8157$ & $8089-8204$ & 39 & 5693-5765 \\
\hline 71 & $7898-8011$ & $7933-8042$ & $7973-8087$ & 40 & $5551-5620$ \\
\hline 72 & $7790-7900$ & 7823-7930 & 7861-7974 & 41 & $5416-5483$ \\
\hline 73 & 7684-7791 & $7715-7822$ & $7752-7863$ & 42 & $5287-5353$ \\
\hline 74 & $7580-7686$ & 7611-7716 & $7646-7755$ & 43 & 5164-5228 \\
\hline 75 & $7480-7584$ & $7510-7613$ & $7543-7651$ & 44 & 5046-5109 \\
\hline 76 & 7381-7484 & 7411-7513 & $7442-7549$ & 45 & 4934-4996 \\
\hline 77 & $7286-7387$ & 7315-7416 & 7345-7449 & 46 & $4827-4887$ \\
\hline 78 & 7192-7292 & 7221-7321 & $7250-7353$ & 47 & $4724-4783$ \\
\hline 79 & $7101-7200$ & $7130-7228$ & $7157-7259$ & 48 & $4626-4684$ \\
\hline 80 & $7012-7110$ & $7041-7138$ & $7066-7167$ & 49 & $4532-4588$ \\
\hline 81 & $6926-7023$ & 6954-7050 & $6978-7077$ & 50 & $4441-4496$ \\
\hline 82 & $6842-6937$ & $6870-6964$ & $6892-6990$ & 51 & $4353-4408$ \\
\hline 83 & $6760-6854$ & $6787-6880$ & 6808-6905 & 52 & $4270-4323$ \\
\hline 84 & $6679-6772$ & $6706-6798$ & $6726-6822$ & 53 & $4189-4241$ \\
\hline 85 & $6601-6693$ & $6628-6719$ & $6646-6741$ & 54 & $4111-4163$ \\
\hline 86 & $6524-6615$ & $6551-6640$ & $6568-6662$ & 55 & $4037-4087$ \\
\hline 87 & $6449-6539$ & $6476-6564$ & 6491-6584 & 56 & $3965-4015$ \\
\hline 88 & $6376-6465$ & $6402-6490$ & $6417-6509$ & & \\
\hline 89 & 6304-6392 & $6330-6417$ & $6345-6435$ & & \\
\hline 90 & 6234-6321 & $6260-6346$ & $6273-6363$ & & \\
\hline 91 & 6166-6252 & $6191-6276$ & 6204-6292 & & \\
\hline 92 & 6099-6184 & 6124-6208 & $6135-6223$ & & \\
\hline 93 & 6034-6118 & $6058-6141$ & 6069-6155 & & \\
\hline 94 & $5970-6053$ & $5994-6076$ & 6004-6089 & & \\
\hline 95 & $5907-5989$ & 5931-6012 & $5940-6025$ & & \\
\hline 96 & $5845-5927$ & 5869-5950 & $5878-5961$ & & \\
\hline 97 & $5785-5866$ & 5809-5888 & 5816-5899 & & \\
\hline 98 & $5727-5806$ & $5750-5829$ & $5757-5839$ & & \\
\hline 99 & $5669-5747$ & $5692-5769$ & $5698-5779$ & & \\
\hline 100 & $5612-5690$ & $5635-5712$ & $5641-5721$ & & \\
\hline 101 & $5557-5634$ & $5579-5656$ & $5585-5664$ & & \\
\hline 102 & $5502-5579$ & $5525-5600$ & 5529-5608 & & \\
\hline 103 & 5471-5546 & $5475-5553$ & & & \\
\hline
\end{tabular}

\section{Discussion}

\section{1. $\mathrm{Na}$}

The velocities and equivalent widths of sodium $D$ emission lines on March 26, 28 and April 22 were measured (see Table 5). Compared with the geocentric radial velocities of the comet, $\dot{\Delta}$, see Table 1 , the nucleocentric velocities are small, less than the error of measurement. The small nucleocentric velocities are expected since the slit was positioned at the center of brightness of the comet.
The sodium emission feature of comets is caused by resonant scattering of the incoming solar light. Therefore, the line intensity and acceleration of sodium atoms by solar radiation pressure depends strongly on the Doppler shift with respect to the solar Fraunhofer absorption lines (Swings effect). Figure 3 shows the comparison of sodium emissions from spectra taken on March 26, 28 and April 22. The intensity of sodium emission lines on April 22 increases about 5 times compared to March 26, 28 , because the solar flux at the excitation wavelength of 
Table 3. A sample of the catalogue of emission lines. In the table, Cols. 1-6 give wavelengths and $E W$ s of emission features on March 26, 28 and April 22, respectively. Columns 7-10 list the laboratory wavelengths of given identification, identified species, bands and transitions, respectively. If the emission features are blended by two or more lines, identified molecules, bands and transitions are listed in subsequent rows. If the emission line is unidentified, Col. 8 states "unid."

\begin{tabular}{|c|c|c|c|c|c|c|c|c|c|}
\hline $\begin{array}{l}\lambda_{26} \\
(\AA)\end{array}$ & $\begin{array}{r}E W_{26} \\
(\mathrm{~m} \AA)\end{array}$ & $\begin{array}{l}\lambda_{28} \\
(\AA)\end{array}$ & $\begin{array}{r}E W_{28} \\
(\mathrm{~m} \AA)\end{array}$ & $\begin{array}{l}\lambda_{22} \\
(\AA)\end{array}$ & $\begin{array}{r}E W_{22} \\
(\mathrm{~m} \AA)\end{array}$ & $\begin{array}{l}\lambda_{\text {lab }} \\
(\AA)\end{array}$ & Species & Band & Transition \\
\hline 5811.29 & 6 & 5811.34 & 6 & & & 5811.28 & $\mathrm{NH}_{2}$ & $(0,10,0)$ & $3_{30}-2_{20}$ \\
\hline 5868.87 & 11 & 5868.80 & 11 & 5868.83 & 7 & 5868.82 & $\mathrm{NH}_{2}$ & $(1,6,0)$ & $3_{30}-4_{40}$ \\
\hline 5871.00 & 11 & 5871.09 & 16 & 5871.07 & 17 & 5871.04 & $\mathrm{NH}_{2}$ & $(1,6,0)$ & $330-440$ \\
\hline 5874.54 & 8 & 5874.58 & 7 & & & $\begin{array}{l}5874.49 \\
5874.50\end{array}$ & $\begin{array}{l}\mathrm{C}_{2} \\
\mathrm{C}_{2}\end{array}$ & $\begin{array}{l}(1,3) \\
(3,5)\end{array}$ & $\begin{array}{l}R_{1}(57)+R_{2}(56)+R_{3}(55) \\
R_{3}(38)\end{array}$ \\
\hline 5890.00 & 260 & 5889.97 & 262 & 5889.95 & 1412 & 5889.97 & $\mathrm{NaI}$ & & \\
\hline 5896.01 & 240 & 5896.00 & 170 & 5895.95 & 774 & 5895.94 & $\mathrm{NaI}$ & & \\
\hline 5909.70 & 14 & 5909.68 & 13 & 5909.63 & 11 & & unid. & & \\
\hline 5915.12 & 18 & 5915.13 & 20 & 5915.15 & 21 & 5915.23 & $\mathrm{C}_{2}$ & $(3,5)$ & $R_{1}(31)+R_{2}(30)$ \\
\hline \multirow[t]{2}{*}{5917.73} & 10 & 5917.68 & 6 & & & 5917.76 & $\mathrm{C}_{2}$ & $(2,4)$ & $R_{1}(41)$ \\
\hline & & & & & & 5917.68 & $\mathrm{C}_{2}$ & $(5,7)$ & $P_{1}(24)$ \\
\hline 5927.99 & 7 & 5928.05 & 14 & & & 5928.06 & $\mathrm{C}_{2}$ & $(2,4)$ & $R_{1}(39)$ \\
\hline 5931.06 & 9 & 5931.02 & 12 & & & 5931.07 & $\mathrm{NH}_{2}$ & $(1,10,0)$ & $2_{20}-1_{11}$ \\
\hline \multirow[t]{2}{*}{5962.68} & 43 & 5962.56 & 48 & & & 5962.61 & $\mathrm{NH}_{2}$ & $(0,9,0)$ & $3_{03}-2_{11}$ \\
\hline & & & & & & 5962.54 & $\mathrm{NH}_{2}$ & $(0,9,0)$ & $3_{03}-2_{11}$ \\
\hline 5965.27 & 14 & 5965.28 & 17 & & & 5965.19 & $\mathrm{NH}_{2}$ & $(0,9,0)$ & $2_{02}-1_{10}$ \\
\hline \multirow[t]{2}{*}{5976.49} & 223 & 5976.41 & 248 & & & 5976.40 & $\mathrm{NH}_{2}$ & $(0,9,0)$ & $1_{01}-1_{11}$ \\
\hline & & & & & & 5976.50 & $\mathrm{NH}_{2}$ & $(0,9,0)$ & $1_{01}-1_{11}$ \\
\hline
\end{tabular}

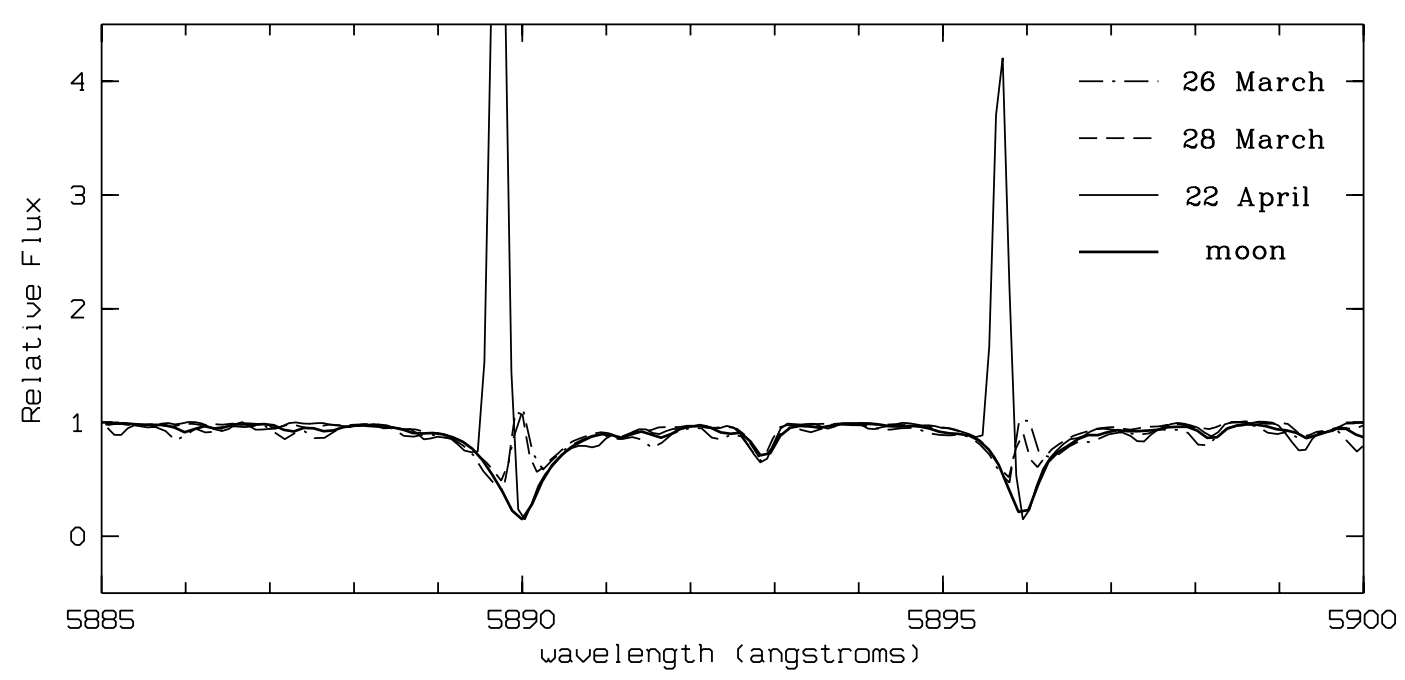

Fig. 3. Comparison of sodium emission on 26, 28 March and 22 April. The cometary continuum is shifted to zero-velocity position. The moon spectrum is also drawn as a reference with the Na D Fraunhofer line

the cometary sodium lines on April 22 is higher than on March 26, 28.

According to calculations of Arpigny et al. (1998), our $\mathrm{Na} D_{2} / D_{1}$ ratio agrees with the model combining resonance scattering and telluric water lines (case 2 in Arpigny et al. 1998). It supports the results of Arpigny et al. (1998).

\section{2. [OI]}

The spectra include $\lambda 5577\left({ }^{1} \mathrm{~S}-{ }^{1} \mathrm{D}\right)$ and the red doublet $\lambda \lambda 6300,6364\left({ }^{1} \mathrm{D}-{ }^{3} \mathrm{P}\right)$ of $[\mathrm{O} \mathrm{I}]$. The intensity ratio, $I_{\lambda 5577} /\left(I_{\lambda 6300}+I_{\lambda 6364}\right)$, is used to determine the parent molecule which dissociates and forms the excited $\mathrm{O}$ atoms. In particular, the dissociation of $\mathrm{H}_{2} \mathrm{O}$ produces roughly ten times as many atoms in the ${ }^{1} \mathrm{D}$ as in the ${ }^{1} \mathrm{~S}$ state and leads to an intensity ratio around 0.10 (Festou et al. 1981). On the other hand, the dissociation of pure $\mathrm{CO}_{2}$ or $\mathrm{CO}$ leads to roughly equal populations in the two states and, to some extent, it contributes to the production of $\mathrm{O}$ with a large intensity ratio. Morrison et al. (1997) and Fink \& Johnson (1984) found that the data are well explained by the dissociation of $\mathrm{H}_{2} \mathrm{O}$ alone.

The average $E W \mathrm{~s}$ for $\lambda \lambda 5577,6300$ and 6364 are $0.032,0.123$ and $0.041 \AA$, respectively. If the blends with 
Table 4. Unidentified lines. In the table, Cols. $1-5$ and 6 give wavelengths and $E W$ s of emission features on March 22,28 and April 22 respectively, Cols. 7 and 8 give wavelengths and relative intensities listed by Brown et al. (1996). Columns 9 and 10 give wavelengths and $E W$ s listed by Morrison et al. (1997)

\begin{tabular}{|c|c|c|c|c|c|c|c|c|c|}
\hline $\begin{array}{l}\lambda_{26} \\
(\AA) \\
\end{array}$ & $\begin{array}{r}E W_{26} \\
(\mathrm{~m} \AA) \\
\end{array}$ & $\begin{array}{l}\lambda_{28} \\
(\AA)\end{array}$ & $\begin{array}{r}E W_{28} \\
(\mathrm{~m} \AA) \\
\end{array}$ & $\begin{array}{l}\lambda_{22} \\
(\AA) \\
\end{array}$ & $\begin{array}{r}E W_{22} \\
(\mathrm{~m} \AA) \\
\end{array}$ & $\begin{array}{l}\lambda_{\mathrm{B}} \\
(\AA) \\
\end{array}$ & $\begin{array}{r}\mathrm{I}_{\mathrm{B}} \\
\text { (Rel.) }\end{array}$ & $\begin{array}{l}\lambda_{\mathrm{M}} \\
(\AA) \\
\end{array}$ & $\begin{array}{r}E W_{\mathrm{M}} \\
(\mathrm{m} \AA)\end{array}$ \\
\hline & & & & 4838.27 & 36 & 4838.20 & 19 & & \\
\hline & & & & 4838.53 & 36 & 4838.40 & 58 & & \\
\hline & & & & 4850.66 & 16 & 4850.62 & 22 & & \\
\hline & & & & 5306.98 & 9 & 5306.91 & 50 & & \\
\hline & & & & 5318.19 & 98 & 5318.15 & 172 & 5318.079 & 381 \\
\hline & & & & 5333.01 & 65 & 5333.04 & 103 & 5332.924 & 212 \\
\hline & & & & 5428.19 & 24 & 5428.15 & 110 & & \\
\hline 5586.15 & 17 & 5586.22 & 16 & 5586.27 & 21 & 5586.18 & 98 & 5586.181 & 99 \\
\hline 5595.35 & 25 & 5595.36 & 27 & 5595.39 & 32 & 5595.36 & 59 & 5595.322 & 101 \\
\hline 5646.08 & 15 & 5646.10 & 8 & & & 5646.06 & 35 & & \\
\hline 5800.90 & 7 & 5800.89 & 5 & & & 5800.75 & 35 & & \\
\hline 5909.70 & 14 & 5909.68 & 13 & 5909.63 & 11 & & & 5909.640 & 81 \\
\hline 6245.17 & 8 & 6245.10 & 9 & & & 6245.05 & 113 & & \\
\hline 6294.85 & 17 & 6294.82 & 11 & & & 6294.83 & 58 & & \\
\hline 6297.33 & 78 & 6297.37 & 82 & & & 6297.17 & 551 & & \\
\hline 6298.75 & 119 & 6298.65 & 128 & & & 6298.66 & 484 & & \\
\hline 6309.60 & 14 & 6309.44 & 8 & & & 6309.46 & 111 & & \\
\hline 6374.16 & 6 & 6374.04 & 6 & 6374.27 & 7 & 6374.15 & 41 & & \\
\hline 6428.80 & 14 & 6428.78 & 11 & & & 6428.74 & 56 & & \\
\hline 6448.15 & 13 & 6448.08 & 12 & & & 6448.04 & 107 & & \\
\hline 6512.46 & 8 & 6512.33 & 24 & & & 6512.37 & 66 & & \\
\hline 6578.19 & 82 & 6578.21 & 81 & & & 6578.11 & 130 & & \\
\hline 6588.10 & 16 & 6587.99 & 16 & & & 6588.01 & 68 & 6587.928 & 66 \\
\hline 6598.84 & 15 & 6598.78 & 12 & & & 6598.83 & 110 & & \\
\hline 6599.43 & 5 & 6599.38 & 8 & & & 6599.26 & 51 & & \\
\hline 6614.41 & 10 & 6614.46 & 11 & & & 6614.39 & 46 & & \\
\hline 6629.52 & 18 & 6629.55 & 10 & & & 6629.50 & 45 & & \\
\hline 6647.92 & 6 & 6647.91 & 7 & & & 6647.89 & 50 & & \\
\hline 6670.58 & 5 & 6670.61 & 6 & & & 6670.53 & 47 & & \\
\hline 6724.39 & 13 & 6724.41 & 12 & & & 6724.42 & 40 & & \\
\hline 6740.91 & 20 & 6740.82 & 20 & & & 6740.83 & 67 & 6740.803 & 55 \\
\hline 6741.73 & 9 & 6741.58 & 10 & & & 6741.60 & 55 & & \\
\hline 6818.85 & 7 & 6818.78 & 8 & & & 6818.75 & 47 & & \\
\hline 6828.11 & 6 & 6828.03 & 5 & & & 6827.99 & 50 & & \\
\hline 6830.94 & 7 & 6830.86 & 6 & & & 6830.87 & 45 & & \\
\hline 6831.89 & 12 & 6831.85 & 14 & & & 6831.84 & 129 & & \\
\hline 6832.48 & 11 & 6832.45 & 11 & & & 6832.48 & 48 & & \\
\hline 6836.61 & 11 & 6836.53 & 11 & & & 6836.47 & 118 & & \\
\hline 6847.34 & 7 & 6847.32 & 7 & & & 6847.22 & 47 & & \\
\hline 6848.64 & 5 & 6848.68 & 8 & & & 6848.59 & 59 & & \\
\hline 6856.08 & 10 & 6856.16 & 16 & & & 6856.09 & 44 & & \\
\hline 6859.38 & 7 & 6859.39 & 6 & & & 6859.31 & 47 & & \\
\hline 6860.91 & 8 & 6860.84 & 6 & & & 6860.81 & 40 & & \\
\hline 6862.20 & 14 & 6862.18 & 15 & & & 6862.08 & 54 & & \\
\hline 6862.45 & 8 & 6862.39 & 9 & & & 6862.31 & 61 & & \\
\hline 6932.30 & 34 & 6932.32 & 35 & & & 6932.32 & 192 & & \\
\hline 6954.84 & 36 & 6954.76 & 25 & & & 6954.77 & 78 & & \\
\hline 6985.52 & 22 & 6985.53 & 19 & & & 6985.51 & 81 & & \\
\hline 7014.67 & 7 & 7014.77 & 6 & & & 7014.68 & 41 & & \\
\hline 7016.67 & 20 & 7016.56 & 42 & & & 7016.63 & 126 & & \\
\hline 7041.81 & 18 & 7041.72 & 38 & & & 7041.68 & 66 & & \\
\hline 7048.30 & 16 & 7048.26 & 19 & & & 7048.22 & 52 & & \\
\hline 7058.21 & 9 & 7058.21 & 16 & & & 7058.09 & 39 & & \\
\hline 7074.38 & 27 & 7074.40 & 25 & & & 7074.36 & 64 & & \\
\hline 7329.49 & 10 & 7329.53 & 12 & & & 7329.48 & 40 & & \\
\hline 7359.70 & 12 & 7359.70 & 15 & & & 7359.70 & 61 & & \\
\hline 7398.17 & 7 & 7398.12 & 13 & & & 7398.06 & 44 & & \\
\hline 7400.29 & 11 & 7400.42 & 18 & & & 7400.42 & 51 & & \\
\hline 7426.96 & 9 & 7426.94 & 8 & & & 7426.83 & 40 & & \\
\hline 7428.38 & 21 & 7428.47 & 18 & & & 7428.42 & 52 & & \\
\hline 7464.84 & 15 & 7464.68 & 13 & & & 7464.68 & 106 & & \\
\hline 7465.31 & 10 & 7465.44 & 7 & & & 7465.37 & 50 & & \\
\hline 7496.34 & 11 & 7496.30 & 9 & & & 7496.23 & 33 & & \\
\hline 7583.07 & 10 & 7583.00 & 8 & & & 7583.00 & 35 & & \\
\hline 7585.31 & 10 & 7585.29 & 11 & & & 7585.30 & 36 & & \\
\hline 7734.11 & 16 & 7733.96 & 16 & & & 7733.96 & 40 & & \\
\hline 7753.70 & 9 & 7753.76 & 11 & & & 7753.64 & 40 & & \\
\hline 7763.50 & 9 & 7763.45 & 9 & & & 7763.47 & 39 & & \\
\hline 7771.05 & 19 & 7771.02 & 17 & & & 7770.97 & 109 & & \\
\hline 7783.08 & 10 & 7783.09 & 9 & & & 7783.03 & 35 & & \\
\hline 7808.81 & 10 & 7808.84 & 6 & & & 7808.73 & 24 & & \\
\hline 7809.63 & 8 & 7809.61 & 10 & & & 7809.62 & 29 & & \\
\hline 7846.10 & 8 & 7846.07 & 12 & & & 7846.10 & 41 & & \\
\hline
\end{tabular}


Table 5. Results of sodium emission lines. The velocities and equivalent width ratio $D_{2} / D_{1}$ of emission lines are listed

\begin{tabular}{lcc}
\hline \hline Date & $\begin{array}{c}\text { Nucleocentric velocities } \\
\left(\mathrm{km} \mathrm{s}^{-1}\right)\end{array}$ & $D_{2} / D_{1}$ \\
\hline 26 March & -0.5 & 1.08 \\
28 March & -0.8 & 1.54 \\
22 April & -2.5 & 1.82 \\
\hline \hline
\end{tabular}

$\mathrm{C}_{2}$ lines at $5577.40 \AA$ and $5577.54 \AA$ are considered, the equivalent width for $\lambda 5577\left({ }^{1} \mathrm{~S}-{ }^{1} \mathrm{D}\right)$ is $0.026 \pm 0.003 \AA$.

We corrected the ratio of normalized line fluxes for the slope of the solar continuous flux, which we took from Allen (1973). The slope of the reflectance spectrum of the comet is unknown. With a wavelength-independent reflectance, the $[\mathrm{OI}]$ intensity ratio $I_{\lambda 5577} /\left(I_{\lambda 6300}+I_{\lambda 6364}\right)$ is 0.22 or 0.18 with the correction for blending of $\lambda 5577$. The ratio is consistent with the production of $\mathrm{O}$ atoms by the dissociation of $\mathrm{H}_{2} \mathrm{O}$, according to the model by Festou et al. (1981).

Acknowledgements. We are grateful to the referee for very helpful comments. We also thank Mr. H. B. Li for his help during the observation and data reduction. The research work is supported by the National Natural Science Foundation of China under grant 19725312 and NKBRSF No. G1999075406.

\section{References}

Allen, C. W. 1973, Astrophysical Quantities, 3rd edition (London, Athlone Press), 172

Arpigny, C., Rauer, H., Manfroid, J., et al. 1998, A\&A, 334, L53

Arpigny, C. 1995, in Laboratory and Astronomical High Resolution Spectra, ASP Conf. Ser., 81, 362

Arpigny, C. 1994, in 50th International Meeting of Physical Chemistry, AIP Conf. Proc., No. 50 (AIP, New York)

Brown, M. E., Bouchez, A. H., Spinrad, H., et al. 1996, AJ, 112,1197

Festou, M. C., Rickman, H., \& West, R. M. 1993, ARA\&A, 5, 37

Festou, M. C., \& Feldman, P. D. 1981, A\&A, 103, 154

Fink, U., \& Johnson, J. R. 1984, AJ, 89, 1565

Hicks, M. D., \& Fink, U. 1996, ApJ, 459, 729

Moore, C. E., Minnaert, M. G. J., \& Houtgast, J. 1966, The Solar Spectrum $2935 \AA$ to $8770 \AA$, National Bureau of Standards Monograph 61, Washington

Morrison, N. D., Knauth, D. C., Mulliss, C. L., et al. 1997, PASP, 109, 676

Osterbrock, D. E., Fullbright, J. P., Martel, A. R., et al. 1996, PASP, 108, 277 